\title{
Eye-tracking Social Desirability Bias
}

Olena Kaminska, Institute for Social and Economic Research, University of Essex, UK

Tom Foulsham, Department of Psychology, University of Essex, UK

Key words: social desirability bias, mode effect, satisficing, eye-tracking, GHQ-12

Abstract

Eye tracking is now a common technique studying the moment-by-moment cognition of those processing visual information. This technique has revealed some aspects of survey responding, but has rarely been applied to different survey modes. Our paper uses an innovative method of realworld eye tracking to provide new evidence about attention to sensitive questions and response scale points, in web, face-to-face and paper and pencil self-administered (SAQ) modes. We link gaze duration to responses in order to understand how respondents arrive at socially desirable or undesirable answers. In particular, we investigate how superficial cognitive processing -satisficing-is related to social desirability bias. The findings show that for questions where positive responses are presented at the top of the scale satisficing is related to social desirability bias in self-completion modes. Yet it does not explain the higher incidence of socially desirable responses in a face-to-face mode. Thus our novel technique and new data can shed light on how social desirability biases arise from deliberate misreporting and/or satisficing, and how these may vary across modes.

Funding acknowledgements: This work was supported by the 2011 University of Essex Interdisciplinary Research Fund for Social Sciences "Understanding Survey Responses through EyeTracking: Mixed-Mode Comparison".

Acknowledgements: The authors are grateful to Gemma Luxton for hours of diligent coding of eyetracking data.

Corresponding author: Olena Kaminska, ISER, University of Essex, Wivenhoe Park, Colchester, CO4 3SQ, UK. Phone: +44 1206 873017. Email: olena@essex.ac.uk

\section{Introduction}

To complete a survey, respondents must go through a series of cognitive processes: reading the question and the response options, recalling something about their life or opinion and deciding on an answer. Eye tracking can provide a measure of these processes. This may be particularly useful in studying mode effect, where the survey materials and their presentation may change the processing involved (Kaminska and Foulsham 2014). In this paper, we begin by describing how eye tracking can be applied across settings, with reference to its development in cognitive psychology. We then use this novel method to examine respondent attention in the context of the social desirability bias.

\section{Eye tracking in multiple settings}

We move our eyes frequently in order to centre the most sensitive part of our visual field on items of interest. Researchers can therefore track these movements and obtain a record of the visual information that is being attended to at any given time. Although in some cases we can shift our attention independently of the eyes, during reading and most everyday activities, what we are looking at is what we are paying attention to and processing at that time (Findlay \& Gilchrist, 2001). 
The first eye-tracking experiments were conducted in the early twentieth century, when it was established that people make rapid, jerky "saccades" to redirect the eye towards words (in text) and objects of interest (in pictures). Decades of subsequent research, with increasingly accurate and less invasive technology, showed how where we look, and when we look there, is affected by both the information in front of us and our cognitive processing. For example, our fixation of words during reading depends on the characteristics of the word (e.g., its length) and our expectations based on grammar and language understanding (see Rayner, 2009, for a review of eye movements in cognitive tasks).

Previously, eye-tracking has been successfully used in psychology, sports science and ergonomics. For example, Hayhoe and Ballard (2005) review studies of eye movements during natural behaviours such as walking and while making tea and sandwiches. The results of these studies emphasize that visual attention is deployed in a highly systematic way and as part of a routine of actions, coordinated to select the most important visual information for each moment in time. Real-world eye-tracking proved a useful tool in studying eye movements in car drivers-who fixate a particular point when steering round a bend (Land, and Lee 1994) - and in sportsmen such as cricketers who are highly skilled at predicting where the ball will bounce (Land and McLeod, 2000).

In a similar way, eye tracking can be used to measure respondents' eye movements while they respond to survey questions (see Galesic and Yan (2011) for a technical description of the method). Since its introduction to survey methodology by Redline and Lankford (2001), followed by Graesser et al (2006) and Galesic et al. (2008), eye tracking has gained popularity as a tool to explore visual attention when responding to a web survey (e.g. Ashenfelter and Hughes, 2011; Kamoen et al., 2011; Lenzner et al., 2011a; Lenzner et al., 2011b; Menold et al., 2011; Romano, and Chen 2011; Neuert and Lenzner, 2015; Greszki, Meyer and Schoen, 2015). Eye-tracking has been so successful in detecting issues with web questionnaires that it is now used by the United States Census Bureau (Ashenfelter and Hughes, 2011; Romano, and Chen 2011) and the German Federal Statistical Office (Federal Statistical Office, 2011) as a pre-testing tool.

The eye-trackers used in previous web studies of survey responding (e.g. the TOBII X500) are not suitable for studying visual attention in modes other than on computer, as they only detect eye movements within a PC monitor. Eye-movements are recorded by an infrared camera which is synchronized with a stimulus on the PC screen via specialized software such that the screen view is known at any point of time when the respondent is looking at it. This requirement, and the restricted range of such eye-trackers, means that they are limited to tracing gazes on the PC screen only. Two previous attempts to extend eye-tracking to SAQ mode (Redline and Lankford, 2001; Potaka, 2007) mounted the questionnaire vertically in front of the participant, as if on a computer monitor. However, the set-up and desk-mounted eye-tracker used in these studies were inflexible (because the camera and participant could not move) and so responses may have been different from a real SAQ mode.

To track eye-movements in survey modes which do not involve a PC and have a natural set up we use an innovative real-world eye-tracker, the SMI HED system (Sensorimotoric Instruments; Teltow, Germany). In essence, this eye-tracker follows a similar principle to the one used with the PC: a camera records eye movements, and a calibration routine (where the respondent looks at a series of specified points on a wall or desk) ensures that features from the eye gaze are mapped onto points in the visual field. The respondent's view is not limited, however, and eye movements can be recorded wherever a respondent may look via a system mounted on a headset that is worn on the respondent's head. Simultaneously another "scene" camera records the view in front of the respondent, and software maps the eye position onto this video at each point in time. Because of the flexibility of this system the exact view in front of each respondent differs and changes as they 
move their head. Additional coding is therefore required if a researcher wants to standardize and compare eye movements and fixations across respondents.

In Psychology mobile eye tracking has been used to track attention while people have real conversations, walk down the street, or search for an office in a hallway (Foulsham, 2015). Furthermore, Foulsham, Walker and Kingstone (2011) compared the eye movements of people walking around the real environment to those of people watching a video on computer and found some important differences. Given the differences between attention on computer and in the real world, one may expect differences in visual attention across survey modes as well.

\section{Experiment}

We next describe the set-up for a novel empirical study, before discussing how our analysis of visual attention can be applied to questions prone to social desirability bias.

The data collection was set up in a Psychology laboratory. We aimed to find the closest set up to a real survey interview while obtaining good-quality eye tracking information. While wearing headsets with the real world eye-tracker, respondents were not restricted in their posture, eye and head movement. No instructions on where to look or how to move / sit were given, except during the set up of the equipment. No person (interviewer or instructor) was present when respondents completed the survey in web or SAQ modes: the respondents were left on their own in the lab after the equipment was set up. Only the interviewer was present during the face-to-face interview.

In total, 20 participants were invited from a list of volunteers for Psychology experiments, with the requirement of having normal vision. The first six respondents assigned to SAQ mode were slouching during the interview such that the data became uncodable. This was resolved by getting respondents to start filling the questionnaire before setting up the eye-tracker. The following two interviews in SAQ mode provided reliable data. In our analysis SAQ and web modes are analysed together as these modes are similar to each other with respect to social desirability bias. Overall, the analysis is based on 9 self-completion interviews (7 web, 2 SAQ) and 5 face-to-face interviews which took place between October 2011 and March 2012.

Of 14 participants with codable data 3 were male ( 2 in face-to-face mode and 1 in web mode), 3 were not native English speakers ( 1 in each mode) and all were between 20 and 26 years old. This is not a representative study and the results should not be treated as such. The strength of the study is in its experimental design in which respondents were randomly assigned to modes, and therefore the measurement error observed is not related to mode choice or self selection into mode.

All questions in face-to-face mode were asked with show cards. The response categories were not read out by an interviewer. In this situation, the respondent's awareness of response options is reliant on their visual perception: seeing and reading each option. The interviewer instructed the respondent to turn over each show card before starting to read the question. No instructions were given on how to hold show cards - respondents were free to hold them, place them on laps or on a desk, keep them sideways or wave them around. While eye-tracking in a web mode is possible with a desk-mounted eye-tracker (e.g. the TOBII X500), which codes visual gazes automatically, we used the real-world eye-tracker for web mode for comparability with other modes.

The eye-tracking information was then manually coded by a coder who watched the resulting videos at a slow speed using custom annotation ChronoViz software (Fouse et al., 2011). The eye tracker recorded eye position at $50 \mathrm{~Hz}$. The general health questionnaire (GHQ-12) (Goldberg and Williams, 1991), which is used broadly in major and small-scale studies to measure general well-being and distress, was selected for coding and analysis. 


\section{Responding to sensitive questions}

Our real-world eyetracking set-up provided an opportunity to investigate cognitive processes during responses made to sensitive questions. Social desirability bias is one of the recognized types of measurement error and occurs when a respondent provides an answer which is more socially acceptable than his / her true attitude or behaviour. Studies that have compared survey reports to external records consistently found underreporting of socially undesirable behaviour, e.g. underreporting abortions or drug use (Schaeffer 2000; and Tourangeau et al. 2007) or overreporting of socially desirable behaviour, e.g. overreporting voting (Anderson and Silver 1986; Belli et al., 2001). This poses a challenge for social methodologists first to understand the causes of social desirability bias, and second to develop the best design methods to improve the reporting of socially undesirable attitudes and behaviours.

The most common source of social desirability bias mentioned in the literature is the respondent's lack of comfort to reveal his or her true attitudes (Tourangeau et al., 2000; Tourangeau et al., 2007; Groves et al., 2009, Holgraves 2004), also called 'impression management' (Holgraves, 2004). Respondents are thought to act so as to avoid the embarrassment, unease and distress that revealing socially undesirable answers may bring. In describing four stages of cognitive processing (comprehension, retrieval, judgement and response) Tourangeau, Rips and Rasinski (2000) suggest that social desirability bias occurs at the last stage of this process: response. This is the stage when respondents map their response onto the scale and may 'edit' it in order to make themselves look more favourable. The theory implies that the respondent has understood the question correctly, retrieved all the relevant information from his/her memory adequately, and has integrated this information well. In other words, the important condition is that the respondent has arrived at the correct answer and is aware of it. Social desirability bias occurs due to purposeful misreporting of this answer at the last stage of the cognitive process.

Much empirical evidence has been gathered which supports the above cause (Lee and Lee, 2012; van Meter, 2005; van Meter, 2000). First of all, a number of experimental studies have consistently shown higher chance of revealing socially undesirable answers in self-completion modes (either SAQ or web) in comparison to interviewer administered modes, especially a face-to-face mode (Holbrook and Krosnick 2010; de Leeuw 2005; Tourangeau et al., 2000; Schwarz et al., 1991; van der Zouwen and de Leeuw, 1990; van der Zouwen and de Leeuw, 1991). It is understood that respondents feel high unease in front of interviewers if their answers do not conform to norms or social expectations, and therefore alter their answer in order to look better in the eyes of the interviewer.

Another set of evidence which may be thought to support the theory of social desirability bias being due to embarrassment comes from studies on questionnaire design. Wording questions in less threatening way has been shown to increase socially undesirable answers (Holbrook and Krosnick 2010; Peter and Valkenburg 2011; Malvin and Moskovitz 1983; Boeije nad Lensvelt-Mulders, 2002; Droitcour and Larson, 2002; Matthews and Velleman, 1997). Such wording may imply that socially undesirable behaviour or attitude exists, and is common, may give excuses for why one may have socially undesirable attitudes or may simply ask more embarrassing questions first such that in light of them the question of interest is perceived as less embarrassing (Barton, 1958). Thus, decreasing embarrassment of a question through wording or question order seems to influence the conscious reporting of true attitudes or behaviour.

The intuitive embarrassment theory of social desirability bias and the above experimental empirical studies have led to clear improvements in how we collect socially sensitive data. It has become common in survey practice to use a careful nonthreatening wording when asking sensitive 
questions, and in surveys conducted face-to-face to collect socially undesirable data via selfadministered mode (CASI, ACASI or SAQ).

A less commonly discussed cause of social desirability is satisficing, a cognitive process that is superficial or not complete. There are two routes of satisficing that potentially can lead to socially desirable answers: desirable answers may be cognitively easier given how they are stored in the memory or how they are retrieved; or socially desirable answers may be selected more frequently due to primacy effect if positive answers are placed at the top of the response scale.

Cognitive ease of socially desirable answers is discussed by Schaeffer (2000), Holgraves (2004) and Belli (2001). Schaeffer suggests that heuristic process (satisficing) can lead to socially desirable answers through a number of ways. First, respondents may retrieve a socially desirable value and use it as an anchor in 'an anchor and adjust response strategy'. Second, respondents may use 'a routine denial' instead of refusing to answer the question, something that can be perceived as socially appropriate in the situation when the person asking an uncomfortable question does not have any right to do so. The author writes (Schaeffer, 2000, p.119): "I would speculate that recipients of intrusive questions routinely use denial as an automatic, socially routine, defensive strategy. Such conventional denials could be offered simply because an intrusive question was asked and without any calculation of risk." Third, the answer may be effortless and ready as a 'public' answer to the question which is part of the respondent's public biography. Schaeffer suggests that respondents may store information at two layers: public information which creates a public image and is easily available; and private information which reflects the truth.

Similarly, Holgraves (2004) outlines two possible ways how responding in a socially desirable way may result from heuristic processing. First, a respondent may simply skip the retrieval stage and provide a response that is only based on socially desirable implications. Second, a respondent may use heuristics during the retrieval stage, mainly by recalling positive information and neglecting negative information. Additionally, Belli et al. (2001) mention that in recalling factual episodes social desirability bias may result from 'source monitoring', when a respondent makes inferences about his/her behaviour during the retrieval process by confusing other similar events with the episode in question. Such selective or incomplete retrieval could also result in a biased response.

Alternatively, in the questions where response scale starts with positive response options, satisficing may lead to social desirability bias through primacy effect. Primacy effect, the tendency to select response options at the top of a response scale in visual modes, has been previously found in questions of non-sensitive nature (Krosnick, 1991; Galesic et al., 2008), and especially for questions with long response option lists of categorical nature, where many categories may be suitable as an answer (Tourangeau et al., 2000; Schwartz et al., 1991). No suggestion has been made in the literature to-date of the primacy effect for sensitive questions, but one may expect primacy effect as a result of 'anchor and adjust response strategy' mentioned by Schaeffer (2000). Response options that are read first may be used as anchors with which the true response may be compared. If response scale starts with positive response options at the top, these options may have higher chance of selection resulting in social desirability bias.

The important distinction between the two causes of social desirability described here is that the embarrassment cause suggests that the respondent has arrived at a correct answer, is aware of it and purposely misreports the answer. In contrast, the satisficing cause suggests that a respondent has not completed adequate cognitive processing, and therefore arrived at a wrong answer. Interestingly, the idea that social desirability bias is caused by both conscious and unconscious factors has been accepted in Psychology for many years (see Paulhus, 2002, for a review). The conscious factor (called 'impression management') is similar to deliberate misreporting due to embarrassment discussed here. The unconscious factor is called 'self-deception' and is similar to the use of 'public answer' or 'routine denial' discussed in the context of satisficing by Schaeffer (2000). 
Over the years there has been much empirical support for conscious and unconscious factors relating to the overall tendency of respondents to provide socially desirable answers (Paulhus, 2002). Yet, there are no empirical studies of satisficing as a cause of social desirability in the context of survey responding to date. Our paper aims to fill this gap.

We aim to test two hypotheses:

1. Is there a relationship between satisficing and social desirability bias in self-completion modes in the situation when positive response options are presented at the top of a vertical scale?

2. Can satisficing explain the difference in social-desirability between face-to-face and selfcompletion modes?

In the next section we use empirical evidence from our laboratory experiment with the real-world eyetracking set-up to address these predictions.

\section{Analysis and results}

The questions selected to the analysis come from a standard GHQ-12 scale. All 12 questions have a 4-point scale where socially desirable response points are presented at the top of the list (see Appendix 1). The scale is of ordinal nature, and its wording changes from questions to question with only three questions having the same scale options as the previous one.

We coded the beginning and end time points when the interviewer or respondent were reading any instructions; when the interviewer or respondent were reading the question; when a respondent was looking at either of the top two (socially desirable) response options; when a respondent was looking at the bottom two (socially undesirable) response options; when a respondent was looking away (at an interviewer / or something else e.g., the wall); and when the respondent's gazes were uncodable. Of all gazes $13-14 \%$ were uncodable in each mode, due to natural eye blinks and tracker data loss. Overall, we obtained time information for 1847 gazes (eye-fixations lasting over 100 milliseconds). For analysis, we combine much of this information to obtain question-level data.

The analysis is conducted at the question level. Given that questions are nested within respondents and respondents may vary in average speed reading and their tendency to look at particular response options, we control for clustering within respondents in all models presented in this paper. Logistic regression with control for clustering within respondents was implemented in Stata 12.1.

\section{Satisficing as a cause of social desirability bias in self-completion mode}

To test whether satisficing is related to the tendency to give socially desirable responses we first explore self-completion modes. Here respondents are free to read and process questions carefully or superficially. Our expectation is that a lack of careful attention to a question will be related to a higher chance of giving socially desirable answers.

From eye-tracking a number of different measures can be obtained, including the time spent reading the question, the time spent looking at socially desirable or undesirable response options, or the time spent on the question overall. Given that time looking at response options may reflect both time reading and selecting a response option, and therefore may be confounded with the option selected, we use the time respondent spent reading the question wording. Question reading usually depends on its number of words. To control for this, we divide the time spent on the question by the number of words (excluding words with fewer than 3 letters, like 'to' and 'on'), thus reflecting the 
average speed of reading per question word. Importantly, the total time spent on reading the question wording is used here, including the first reading of the question combined with all the following re-readings (over $2 / 3$ of questions received at least one rereading). We do not believe that satisficing is linearly related to question reading speed. It is more likely that those questions which are skimmed faster than usual are read with satisficing. We therefore create an indicator of whether a question is read faster than average within self-completion modes or not. In our dataset this corresponds to a reading speed of 400 milliseconds or less per word. Shorter reading speed reflects less attention paid to the question wording. Thus we use this indicator as a proxy measure of satisficing. Readings were not coded for 11 questions, which were excluded from the analysis in this section.

It is of interest to know whether fast reading is related to some particular questions or particular respondents. We find that each question got at least one fast reading, and the number of fast readings ranges between 2 and 6 of 9 readings per question. More variability is observed among respondents. Of the 9 respondents, 2 read all but 1 question carefully, and 2 read over 10 of 12 questions fast, while others read between 3 and 9 questions fast. The two respondents who are nonnative English speakers did not read slower than others (with 3 and 9 questions read fast); and t-test of average word speed within a question showed no significant difference between native and nonnative speakers. Fast reading is therefore not restricted to particular questions or particular respondents and we proceed with the analysis of interest.

Importantly, our analysis is at the question level, where questions are clustered within respondents. We use logistic regression with socially desirable responses as an outcome (socially desirable answer $=1$; socially undesirable answer $=0$ ), and reading faster than $400 \mathrm{~ms}$ per word (fast reading) as an explanatory variable. Results, presented in Table 1 (Model 1), indicate that the relationship between fast reading and socially desirable responses is significant. Questions read faster than $400 \mathrm{~ms}$ per word have 4.2 times higher odds of getting socially desirable answer than questions read slower.

Table 1. Socially desirable answers as a function of fast question reading in self-completion mode

Model 2: questions

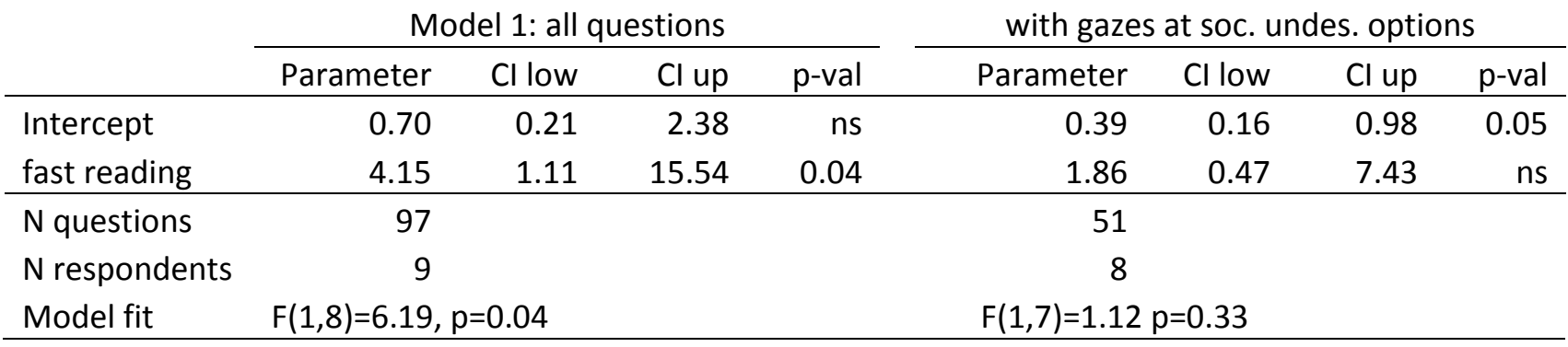

*the parameters reported are odds for the intercept and odds ratios for the explanatory variable

As discussed earlier there are two routes via which satisficing may lead to social desirability bias: cognitive ease of socially desirable answer and primacy effect. To test whether primacy effect is responsible for social desirability answers we next restrict our analysis to only those questions during which a socially undesirable answer (presented at the bottom of the scale) is fixated at least once. The fixation time is selected to be at least $200 \mathrm{~ms}$, which is a low estimate for the average fixation duration during reading (Rayner, 1998). Of 97 questions 51 got fixations on socially undesirable options. 
When analysis is restricted to these, the relationship between fast question reading (less than $400 \mathrm{~ms}$ per word) and selecting socially desirable responses becomes nonsignificant (Table1, Model 2). This indicates that satisficing effect on social desirability bias is at least partially explained by primacy effect: a respondent reads a question fast, pays most attention to first options, and therefore selects socially desirable answer. Nevertheless, the effect (odds ratio of 1.86) is still present and the lack of significance may be due to a small sample size. Thus, there is still a possibility that satisficing effect on social desirability bias has both routes: through primacy effect and through cognitive ease of positive answers.

\section{Satisficing as a cause of social desirability bias in different modes}

Similar to previous studies (see reviews in de Leeuw 2005; Tourangeau et al., 2000) we find significantly higher levels of socially desirable responses in face-to-face mode than in self-completion modes (Table 2, Model 3). One expectation is that this discrepancy is due to higher embarrassment in face-to-face mode. An alternative explanation, given the results from the previous section, is that the difference may be caused by higher satisficing in face-to-face mode. This explanation, although unlikely, has not been tested empirically before. This section explores whether the difference in social desirability bias between face-to-face and self-completion modes can be explained, at least in part, by satisficing theory.

Table 2. Socially desirable responses depending on the mode of interview

Model 4: questions

Model 3: all questions with gazes at soc undes. options

\begin{tabular}{|c|c|c|c|c|c|c|c|c|}
\hline & \multicolumn{4}{|c|}{ 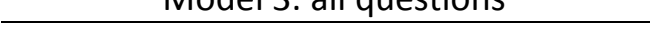 } & \multicolumn{4}{|c|}{ (1) } \\
\hline & Parameter & $\mathrm{Cl}$ low & $\mathrm{Cl}$ up & $p$-val & Parameter & $\mathrm{Cl}$ low & $\mathrm{Cl}$ up & $p$-val \\
\hline Intercept & 1.63 & 0.56 & 4.74 & $\mathrm{~ns}$ & 0.74 & 0.21 & 2.66 & ns \\
\hline face-to-face & 6.73 & 1.35 & 33.46 & 0.02 & 12.38 & 2.31 & 66.48 & 0.01 \\
\hline $\mathrm{N}$ questions & 168 & & & & 112 & & & \\
\hline $\mathrm{N}$ respondents & 14 & & & & 13 & & & \\
\hline Model fit & $F(1,13)=6.6$ & $=0.02$ & & & $F(1,12)=10$ & $p=0.01$ & & \\
\hline
\end{tabular}

*the parameters reported are odds for the intercept and odds ratios for the explanatory variable

Unlike question reading in self-completion mode, the time during which the question is read in faceto-face mode does not depend only on the respondent, but on the respondent-interviewer interaction. This is therefore a less appropriate measure of respondent's satisficing. Yet, if satisficing is higher in face-to-face mode we would observe fewer gazes at the options in the end of the response list. Given that these options are socially undesirable, less attention to them could explain less likelihood of their selection. Recall, interviewers did not read out response options - response options were available only on show cards. Thus respondents in both modes were relying only on visual communication channels for response options. We find that socially undesirable options are looked at in more questions in face-to-face mode than in self-completion modes (Table 3). 
Table 3 Questions with gazes on socially undesirable answers as a function of mode

\begin{tabular}{lrrrr} 
& Parameter & Cl lower & Cl upper & $\mathrm{p}$-value \\
\hline Intercept & 1.30 & 0.45 & 3.72 & $\mathrm{~ns}$ \\
face-to-face & 4.37 & 1.05 & 18.19 & 0.04 \\
\hline N questions & 168 & & & \\
N respondents & 14 & & & \\
Model fit & $\mathrm{F}(1,13)=4.98, \mathrm{p}=0.04$ & & \\
\hline
\end{tabular}

*the parameters reported are odds for the intercept and odds ratios for the explanatory variable

And restricting the analysis to those questions that feature a gaze of at least $200 \mathrm{~ms}$ on socially undesirable answers does not change the conclusion: selecting socially desirable answers is still significantly more likely in face-to-face mode than in self-completion modes (Table 2, Model 4). This contradicts the suggestion that face-to-face mode achieves more social desirable answers because of satisficing. In fact, the results support the alternative theory of embarrassment as a dominant source of social desirability bias in a face-to-face mode.

Finally, we test whether the duration of gaze on socially undesirable response options may explain the difference in responses between modes. Our findings indicate no significant difference in average time respondents fixate at socially undesirable options between face-to-face and selfcompletion modes among the questions that receive such gazes.

Table 4 Total gaze duration on socially undesirable options (in milliseconds) as a function of mode among question that received such gazes

\begin{tabular}{lrrrr} 
& Gaze time & Cl lower & Cl upper & p-value \\
\hline Intercept & 1614.76 & 647.60 & 2581.91 & 0.003 \\
face-to-face & 279.36 & -766.49 & 1325.21 & $\mathrm{~ns}$ \\
\hline N questions & 112 & & & \\
N respondents & 12 & & & \\
Model fit & $\mathrm{F}(1,12)=0.34, \mathrm{p}=0.57$ & & \\
\hline
\end{tabular}

Overall, we do not seem to find any evidence of higher satisficing in face-to-face mode than in selfcompletion modes. If anything, we find evidence of the opposite: respondents are more likely to read socially undesirable answers in face-to-face mode than in other modes. While we cannot show that social desirability bias observed in face-to-face mode is due to embarrassment, we can conclude that it is unlikely a result of satisficing. And therefore, another source, very possibly embarrassment, must be causing the difference in social desirability bias between interviewer- and self-administered modes.

\section{Discussion}

For many years improvements in design for questions prone to social desirability bias tackled embarrassment and conscious misreporting. Much research has explored how to decrease embarrassment through either changing question wording or question context, or via switching to self-administered mode. Yet, causes of social desirability bias, aside of embarrassment, have not received much research attention in questionnaire design until now. 
Our paper provides first empirical findings suggesting that social desirability bias may also occur as a result of satisficing. Specifically, we find that in a self-completion mode faster question reading is linked to a higher chance of giving socially desirable answers. This phenomenon is, at least partially, explained by primacy effect: for questions with response scale starting with positive options, respondents who read questions fast, pay more attention to top response options and select an option among these. An additional route of satisficing leading to social desirability bias is the cognitive ease of providing a positive answer.

This finding is important especially as it opens new ways of improving reports to sensitive questions in self-completion mode. In order to avoid primacy effect leading to socially desirable answers we can reverse the scale such that the top options are of negative, socially undesirable nature. In this situation a respondent should consider the most negative options first before considering cognitively easier, positive ones.

The set of results from mode comparison confirms previous thinking: less satisficing is found in faceto-face mode; and social desirability bias which occurs in face-to-face mode in higher rate than in self-completion mode is found to not be caused by satisficing. Face-to-face mode is often described as a high quality mode due, in part, to the presence of many communication channels: verbal and nonverbal, aural and visual. Interestingly, even when respondents rely only on visual channel for perceiving response options face-to-face mode achieves more looks at bottom categories than selfcompletion mode. Even more interestingly, once a respondent fixates a bottom category the look seems to have a similar duration in face-to-face mode as in self-completion modes. But full perception of a scale in face-to-face mode, and as a result more frequent looks at socially undesirable options, does not explain higher social desirability bias in this mode. An alternative cause, most likely embarrassment, must be present in the face-to-face mode to explain the mode difference.

We would like to caution the reader from simplifying our results in a way that self-completion modes receive socially desirable answers because of satisficing and face-to-face mode because of embarrassment. This is unlikely to reflect the full process leading to socially desirable responses. While we are limited to showing satisficing effects on social desirability bias within self-completion mode, and face-to-face mode featuring less satisficing than self-completion modes, this does not mean that only one source is responsible for social desirability bias in each mode. It is more likely that social desirability bias is influenced by embarrassment and satisficing in both face-to-face and self-completion modes, but this influence may vary in its extent: face-to-face mode is likely to have less social desirability bias due to satisficing but more social desirability bias due to embarrassment in comparison to self-completion modes.

Overall, the results from our study should be treated with caution due to its lack of representation of the general population. The participants were members of a volunteer list for psychological experiments in one university. Although much was done to make the survey situation realistic, the interviews were conducted in a Psychology lab with respondents wearing a helmet with an eyetracker. This all calls for further research into the topic, and only through consistency of the findings can we extrapolate the results more generally.

Finally, our paper provides an example of real-world eye-tracker usage in the context of survey methodology. This is the first usage of such method in this field, which proved useful, especially in the mode comparison area. Future research can study the use of show cards, and visual attention to an interviewer depending on interviewer behaviour / training in a face-to-face mode; the effect of visual lay-out of questions, and response options and question sets in SAQ mode; and off-monitor attention in web mode. Understanding the causes of differences in attention across modes may prove exceptionally valuable for developing mode-proof survey questions in the future. We envisage and encourage further usage of real-world eye-tracking to improve survey quality. 


\section{References}

Anderson, Barbara A. and Brian D. Silver. 1986 "Measurement and Mismeasurement of the Validity of the Self-Reported Vote." American Journal of Political Science 30: 771-785.

Ashenfelter, Kathleen T., and Todd R. Hughes. 2011. "Results from Iterative Usability Testing of the American Community Survey (ACS)." Paper presented at European Survey Research Association Conference, Lausanne, Switzerland

Barton, Allen H. 1958. "Asking the Embarrassing Questions." Public Opinion Quarterly. 22: 67-68.

Belli, Robert F., Michael W. Traugott, and Matthew N Beckmann. 2001. "What Leads to Voting Overreports? Contrasts of Overreporters to Validated Voters and Admitted Nonvoters in the American National Election Studies." Journal of Official Statistics. 17: 479-498.

Boeije, Hennie, and Gerty Lensvelt-Mulders. 2002. "Honest by Chance: a Qualitative Interview Study to Clarify Respondents' (Non-)Compliance with Computer-Assisted Randomized Response." Bulletin de Mèthodologie Sociologique. 75: 24-39.

Droitcour, Judith A., Eric M. Larson. 2002. "An Innovative Technique for Asking Sensitive Questions: the Three-Card Method." Bulletin de Mèthodologie Sociologique. 75: 5-23.

Federal Statistical Office. 2011. Methods - Approaches - Developments: Information of the German Federal Statistical Office. Wiesbaden: Federal Statistical Office.

Foulsham, T., Walker, E. \& Kingstone, A. 2011. "The where, what and when of gaze allocation in the lab and the natural environment." Vision Research. 51(17): 1920-1931.

Fouse, A., N. Weibel, E. Hutchins and J. Hollan. 2011. "ChronoViz: A system for supporting navigation of time-coded data" In Extended Abstracts of CHI 2011, SIGCHI Conference on Human Factors in Computing Systems, Vancouver, Canada, May 2011.

Galesic, Mirta, Roger Tourangeau, Mick P. Couper, Frederick G. Conrad. 2008. "Eye-tracking Data: New Insights on Response Order Effects and Other Cognitive Shortcuts in Survey Responding." Public Opinion Quarterly, 72(5): 892-913

Galesic, Mirta and Ting Yan. 2011. "Use of Eye Tracking for Studying Survey Response Processes." In Social and Behavioral Research and the Internet: Advances in Applied Methods and Research Strategies, eds. Marcel Das, Peter Ester and Lars Kaczmirek. London: Routledge Taylor and Francis Group.

Goldberg D, Williams P. 1991. A User's Guide to the General Health Questionnaire. London: NFERNelson.

Graesser, Arthur C., Zhiqiang Cai, Max M. Louwerse, and Frances Daniel. 2006. "Question Understanding Aid (QUAID): A Web Facility that Tests Question Comprehensibility." Public Opinion Quarterly. 70:1-20

Greszki, Robert, Marco Meyer, Harald Schoen. 2015. "Exploring the Effects of Removing 'Too Fast' Responses and Respondents from Web Surveys." Public Opinion Quarterly. 79: 471-503.

Groves, Robert M., Floyd J. Fowler, Jr., Mick P. Couper, James M. Lepkowski, Eleanor Singer, and Roger Tourangeau. 2009. Survey Methodology. Hoboken, New Jersey: John Wiley and Sons, Inc.

Hayhoe, Mary, and Dana Ballard. 2005. "Eye movements in natural behaviour." Trends in Cognitive Sciences. 9(4): 188-194

Holbrool, Allyson, and Jon. A. Krosnick. 2010. "Social Desirability Bias in Voter Turnout Reports: Tests using the Item Count Technique." Public Opinion Quarterly 74:37-67. 
Holgraves, Thomas. 2004. "Social Desirability and Self-Reports: Testing Models of Socially Desirable Responding." Personality and Social Psychology Bulletin. 30: 161-172.

Kaminska, Olena, and Tom Foulsham. 2014. "Real-world Eye-tracking in Face-to-faceand Web Modes." Journal of Survey Statistics and Methodology: 2014(2): 343-359

Kamoen, Naomi, Bregje Holleman, Pim Mak, and Ted Sanders. 2011. "Agree or Disagree? Cognitive Processes in Answering Contrastive Survey Questions." Paper presented at European Survey Research Association Conference, Lausanne, Switzerland

Krosnick, Jon A. 1991. "Response Strategies for Coping with the Cognitive Demands of Attitude Measures in Surveys." Applied Cognitive Psychology. 5: 231-236.

Land M F, Lee D N. 1994. "Where we look when we steer." Nature (London) 369, 742-744

Land, M.F., and P. McLeod. 2000. "From eye movements to actions: how batsmen hit the ball." Nat. Neurosci. 3: 1340-1345.

Lee, Yeon-Ok, Raymond M. Lee. 2012. "Methodological Research on 'Sensitive' Topics: a Decade Review." Bulletin de Mèthodologie Sociologique. 114: 35-49.

de Leeuw, Edith D. 2005. "To Mix or Not to Mix Data Collections Modes in Surveys." Journal of Official Statistics. 21: 233-255.

Lenzner, Timo, Lars Kaczmirek, and Mirta Galesic. 2011a. "Seeing Through the Eyes of the Respondent: An Eye-tracking Study on Survey Question Comprehension." International Journal of Public Opinion Research: 23(3): 361-373.

Lenzner, Timo, Lars Kaczmirek, Mirta Galesic, and Mona Merkert. 2011b. "Left feels right! The optimal position of answer boxes in Web surveys." Paper presented at European Survey Research Association Conference, Lausanne, Switzerland

Malvin, Janet H. and Joel Moskowitz. 1983 "Anonymous Versus Identifiable Self-Reports of Adolescent Drug Attitudes, Intentions, and Use." Public Opinion Quarterly 47: 557-566.

Matthews, Zoe, Richard Velleman. 1997. "New Age' travellers, Urban Slum Dwellers, Aborogines and Drug Users: Experiences of Collecting Sensitive Data from Marginalised Communities." Bulletin de Mèthodologie Sociologique. 57: 65-85.

Menold, Natalja, Lars Kaczmirek, and Timo Lenzner. 2011. "Cognitive Processes in Answering Questions in Web surveys: How do Respondents Attend to Verbal Labels in Rating Scales?" Paper presented at European Survey Research Association Conference, Lausanne, Switzerland

Van Meter, Karl M. 2000. "Sensitive Topics - Sensitive Questions: Overview of the Sociological Research Literature." Bulletin de Mèthodologie Sociologique. 68: 59-78.

Van Meter, Karl M. 2005. "Studying Survey Interviewers: a Call for Research and an Overview ." Bulletin de Mèthodologie Sociologique. 87: 51-71.

Neuert, Cornelia E., Timo Lenzner. 2015. "A Comparison of Two Cognitive Pretesting Techniques Supported by Eye-Tracking." Social Science Computer Review 1-15.

Paulhus, Delroy L. "Socially Desirable Responding: the Evolution of a Construct." In The role of constructs in psychological and educational measurement, eds. H.I. Braun, D.N. Jackson, and D. E. Wiley. Mahwah NJ: Erlbaum.

Peter, Jochen and Patti M. Valkenburg. 2011 "The Impact of 'Forgiving' Introductions on the Reporting of Sensitive Behaviour in Surveys." Public Opinion Quarterly 75: 779-787. 
Potaka, Lyn. 2007. "What the Eye doesn't see: A Feasibility Study to Evaluate Eye-Tracking Technology as a Tool for Paper-Based Questionnaire Development." Proceedings from Quest 2007 Statistics Canada, Ottawa, Canada: 162-171.

Rayner, K. 1998. "Eye Movements in Reading and Information Processing: 20 Years of Research." Psychological Bulletin. 124: 372-422.

Redline, Cleo D., and Christopher P. Lankford. 2001. "Eye-Movement Analysis: A New Tool for Evaluating the Design of Visually Administered Instruments (Paper and Web)." Proceedings of the Survey Research Methods Section of the American Statistical Association, available at www.amstat.org/Sections/Srms/Proceedings/y2001/Proceed/00248.pdf.

Romano, Jennifer C. and Jennifer M. Chen. 2011. "A Usability and Eye-tracking Evaluation of Four Versions of the Online National Survey of College Graduates (NSCG): Iteration 2." Study Series: Survey Methodology 2011-01. Washington D.C.: U.S. Census Bureau.

Schaeffer, Nora Cate. 2000. "Asking Questions About Threatening Topics: A Selective Overview." In The Science of Self-Report: Implications for Research and Practice, eds. Arthur A. Stone, Jaylan S. Turkkan, Christine A. Bachrach, Jared B. Jobe, Howard S. Kurtzman and Virginia S. Cain. London: Lawrence Erlbaum Associates, Publishers.

Schwarz, Norbert, Fritz Strack, Hans-J. Hippler, and George Bishop. 1991. "The Impact of Administration Mode on Response Effects in Survey Measurement." Applied Cognitive Psychology. 5: 193-212.

Tourangeau, R., Rips, L. J., \& Rasinski, K. A. 2000. The psychology of survey response. Cambridge, United Kingdom: Cambridge University Press.

Tourangeau, Roger and Ting Yan. 2007 "Sensitive Questions in Surveys." Psychological Bulletin. 133: 859-883.

Van der Zouwen, Johannes, Edith D. de Leeuw. 1990. "The Relationship between Mode of Administration and Quality of Data in Survey Research 1." Bulletin de Mèthodologie Sociologique. 29: 3-14.

Van der Zouwen, Johannes, Edith D. de Leeuw. 1991. "The Relationship between Mode of Administration and Quality of Data in Survey Research: (final part) 2." Bulletin de Mèthodologie Sociologique. 31: 49-60. 
Appendix 1. GHQ questionnaire

The next questions are about how you have been feeling over the last few weeks.

Have you recently been able to concentrate on whatever you're doing?

- Better than usual

- Same as usual

- Less than usual

- Much less than usual

Have you recently lost much sleep over worry?

- Not at all

- No more than usual

- Rather more than usual

- Much more than usual

Have you recently felt that you were playing a useful part in things?

- More so than usual

- Same as usual

- Less so than usual

- Much less than usual

Have you recently felt capable of making decisions about things?

- More so than usual

- Same as usual

- Less so than usual

- Much less capable

Have you recently felt constantly under strain?

- Not at all

- No more than usual

- Rather more than usual

- Much more than usual

Have you recently felt you couldn't overcome your difficulties?

- Not at all

- No more than usual

- Rather more than usual

- Much more than usual

Have you recently been able to enjoy your normal day-to-day activities?

- More so than usual

- Same as usual

- Less so than usual

- Much less than usual 
Have you recently been able to face up to problems?

- More so than usual

- Same as usual

- Less able than usual

- Much less able

Have you recently been feeling unhappy or depressed?

- Not at all

- No more than usual

- Rather more than usual

- Much more than usual

Have you recently been losing confidence in yourself?

- Not at all

- No more than usual

- Rather more than usual

- Much more than usual

Have you recently been thinking of yourself as a worthless person?

- Not at all

- No more than usual

- Rather more than usual

- Much more than usual

Have you recently been feeling reasonably happy, all things considered?

- More so than usual

- About the same as usual

- Less so than usual

- Much less than usual 